\title{
Effect of Backing on Carbon-Polymer Nanocomposite Sources for Laser Generation of Broadband Ultrasound Pulses
}

\author{
Srinath Rajagopal \\ Ultrasound and Underwater Acoustics \\ National Physical Laboratory \\ Teddington, U.K. \\ srinath.rajagopal@npl.co.uk
}

\author{
Bradley E. Treeby \\ Department of Medical Physics and \\ Biomedical Engineering \\ University College London \\ London, U.K. \\ b.treeby@ucl.ac.uk
}

\author{
Ben T. Cox \\ Department of Medical Physics and \\ Biomedical Engineering \\ University College London \\ London, U.K. \\ b.cox@ucl.ac.uk
}

\begin{abstract}
Light absorbing polymer nanocomposites have been widely investigated as a source of broadband and high amplitude ultrasound generated via pulsed laser excitation. Biomedical applications of such sources include both imaging and ablative therapies. Fabrication of light absorbing nanocomposite sources has mostly involved elaborate chemistry. Here, a nanocomposite composed of multi-walled carbon nanotubes mechanically dispersed in polydimethylsiloxane polymer is used. The nanocomposite film was backed on optically clear polydimethylsiloxane and laboratory grade glass slides. The two backed sources were investigated using two lasers whose full-width half maximum durations were 2.6 and $4.0 \mathrm{~ns}$. It was found that the characteristics of the acoustic field generated from the nanocomposite source showed a significant dependence on the backing when the stress confinement criterion is not satisfied.
\end{abstract}

Keywords-hydrophone, calibration, nanocomposites, photoacoustics

\section{INTRODUCTION}

Laser generation of ultrasound via pulsed laser excitation of polymer nanocomposites and metal nanostructures has been broadly investigated in applications such as all optical ultrasound imaging devices [1]-[3], ablative therapies [4], [5], acoustic kinoforms [6] and more recently as a potential source for calibrating hydrophones [7]. Several nanocomposite materials have been investigated in this regard and we refer the interested reader to [7]-[9] and to the references contained therein.

In a previous study [7], several carbon-polymer nanocomposite (CPN) sources were fabricated involving epoxy, polyurethane and polydimethylsiloxane polymers and multi-walled carbon nanotubes of different weight percentages dispersed within each of the three polymers. Nanocomposite sources were backed on a laboratory grade glass slide. The sources were tested as a function of composite thickness and laser fluence as well as stability over an extended period of laser excitation. The aim of that study was to identify a suitable and stable CPN material for the generation of high pressure, broadband and planar ultrasound source for application in calibration of medical hydrophones [10]. The advantages of such a CPN source are that a single ultrasound pulse spans the measurement bandwidth of most hydrophones and, unlike piezoelectric transducers, high-pressure levels can be achieved without focusing, which ensures errors due to spatial-averaging are small. Additionally, the high signal-to-noise lowers the uncertainty in the measurement.
This paper describes the results of an initial study into the effect of different backings and different laser-pulse durations on the characteristics of the acoustic field generated.

\section{MATERIALS AND METHODS}

\section{A. Carbon-Polymer Nanocomposites}

The multi-walled carbon nanotubes (CNT), polydimethylsiloxane (PDMS) and catalyst (curing agent) were all combined by their weight percentages. A 2.5 weight percent (wt\%) CNT was dispersed in PDMS using shear mixing at $3500 \mathrm{rpm}$ for $2 \mathrm{~min}$ (DAC $150.1 \mathrm{FV}-\mathrm{K}$, SpeedMixer $^{\mathrm{TM}}$, High Wycombe, U.K.). The catalyst was then added, and shear mixed again at $3500 \mathrm{rpm}$ for $2 \mathrm{~min}$. The CNTs were carboxyl functionalized (Haydale Ltd., Ammanford, U.K.). The glass-backed CPN source was fabricated by coating a thin film of the CNT-PDMS mixture on a laboratory-grade glass slide followed by oven curing the thin film at $100{ }^{\circ} \mathrm{C}$ for 35 mins. A blade film applicator (Sheen Instruments, West Molesey, U.K) was used to coat the thin film on the flat surface of a glass slide [7]. The thickness of the cured CPN film on the glass slide was estimated by measuring the thicknesses of the uncoated glass slide and glass slide plus cured CPN film using a $1 \mu \mathrm{m}$ resolution micrometer.

To fabricate the PDMS-backed CPN source the following approach was undertaken. A small amount of petroleum jelly was coated on a parallel glass window (FSW18, Newport Spectra-Physics Ltd, Didcot, U.K.) and then wiped off using lens cleaning tissue. A thin film of the CNT-PDMS mixture was applied on top of the petroleum jelly coated glass window using the blade film applicator described above. The glass window was then placed in a polytetrafluoroethylene mould with coated side facing up and subsequently the mould was filled with a 5:1 ratio of PDMS:catalyst, which is an optically clear mixture. The mould was placed in an oven at $100{ }^{\circ} \mathrm{C}$ for 35 mins. During curing, the presence of petroleum jelly prevents bonding of the CPN film to glass and instead it bonds with PDMS-catalyst mixture forming a PDMS-backed source. Various techniques of PDMS-PDMS bonding are discussed elsewhere [11]. After cooling the mould, the PDMS-backed source was removed and cut to the required lateral dimensions for testing. Glass and PDMSbacked sources are shown in the inset of Fig.1. Four glassbacked and four PDMS-backed sources were fabricated.

As a PDMS backing of unspecified thickness and the CPN film were cured together and the thickness of the CPN film on PDMS couldn't be directly estimated, its thickness 
was derived indirectly. The wavelength-dependent optical absorbance, $A(\lambda)$, of the glass-backed and PDMS-backed sources were measured using a spectrophotometer (400-900 nm, Perkin Elmer, Waltham, MA, USA). The optical absorbances of CPN sources were measured, three times each, near to the central region of the coating over an area of $5 \times 5 \mathrm{~mm}$. The optical absorption coefficient, $\mu_{\mathrm{a}}(\lambda)$ of glassbacked sources was determined using the relation: $\mu_{\mathrm{a}}(\lambda)=$ $A(\lambda) \cdot \ln (10) / d$, where $d$ is the measured thickness of the CPN film coated on glass. By knowing $\mu_{\mathrm{a}}(\lambda)$ of the CPN material, the thickness of the CPN film on PDMS was estimated using the above relation. The differences in the optical properties of the glass slide and PDMS are relatively small. Tthe optical refractive index of the glass and PDMS are 1.47 and 1.4, respectively. Optical absorption coefficients are also similar, hence no corrections were applied to the derived thickness.

\section{B. Experimental Setup}

The experimental setup used to measure the LGUS responses from CPN sources is shown in Fig.1. Two pulsed laser sources both operating at $1064 \mathrm{~nm}$ were employed for the study. The first laser was a Nano 120-S (Litron Lasers Ltd, Rugby, U.K.) with a full-width half-maximum (FWHM) of $4 \mathrm{~ns}$ and a peak energy of $120 \mathrm{~mJ}$ per pulse. The second was a fiber-coupled (Ceramoptec $\mathrm{GmbH}$, Bonn, Germany) M-Nano (Montfort Laser GmbH, Götzis, Austria) laser with a FWHM of $2.6 \mathrm{~ns}$ and a peak energy of $55 \mathrm{~mJ}$ per pulse at the fiber end. The laser energy on the Nano 120 -S was controlled by an in-built motorized $\lambda / 2$ waveplate and polarizing beam splitter and for M-Nano it was controlled by an external manual $\lambda / 2$ waveplate and a polarizing beam splitter (not shown in Fig.1). The output beams from both lasers were homogenized using a 1500 grit ground glass optical diffuser (ODI). The homogenized beam was weakly focused using a plano-convex lens of $150 \mathrm{~mm}$ focal length to reduce the losses due to scatter caused by the ODI. A membrane hydrophone (UC1604, Precision Acoustics Ltd, Dorchester, U.K) with a nominal element diameter of $0.4 \mathrm{~mm}$ was used to measure the LGUS response. The hydrophone's calibration was traceable to National Physical Laboratory's primary standard from 1 to $60 \mathrm{MHz}$ and was also extrapolated from 61 to $110 \mathrm{MHz}$ using a $1 \mathrm{D}$ analytical model [12]. The measured hydrophone voltage time-series from the CPN sources were converted to pressure time-series via deconvolution [13]. The peak-positive pressure and -20

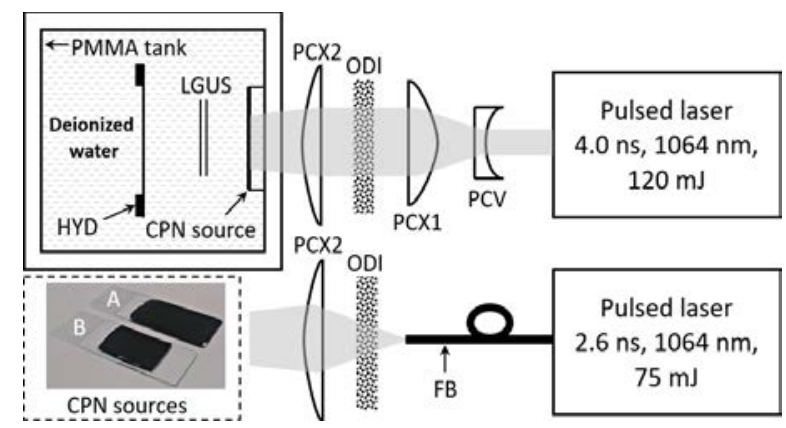

Fig. 1. Top view of the test setup. Plano-concave lens (PCV); planoconvex lens (PCX1 and PCX2); optical diffuser (ODI); fiber-bundle (FB); carbon-polymer nanocomposite (CPN); hydrophone (HYD); laser generated ultrasound (LGUS); Polymethyl methacrylate (PMMA); glass-backed source (A); PDMS-backed source laid on glass slide (B).
$\mathrm{dB}$ bandwidth was computed for each pressure time-series. Further details of the experimental setup can be found in [7].

\section{The Test Protocol}

As two different laser sources were used, M-Nano (2.6 ns) and Nano 120-S (4.0 ns) lasers, the laser energy must be the same for both to allow a comparison of the effect of laser pulse duration on the CPN sources. Also, the LGUS field on the surface of the hydrophone must be sufficiently planar to ensure spatial averaging does not play a role. The planarity was determined by raster scanning the LGUS field generated from the two laser sources using a glass-backed source. An uncalibrated membrane hydrophone (UT1602, Precision Acoustics Ltd, Dorchester, U.K) with a nominal element diameter of $0.2 \mathrm{~mm}$ was used to scan an area of $1.5 \times 1.5 \mathrm{~cm}$ at $0.25 \mathrm{~mm}$ step size at an axial distance of $3.7 \mathrm{~mm}$. Identical laser energies on each CPN surface was ensured as follows. First, the responses from glass and PDMS-backed sources were measured using the $2.6 \mathrm{~ns}$ laser at four rotational positions of the $\lambda / 2$ waveplate, which corresponded to laser energies of $6,9,12$ and $16 \mathrm{~mJ}$. The laser energy was measured using a $20 \mathrm{~mm}$ diameter pyroelectric sensor (ES220C, Thorlabs, Ely, U.K.). Second, the response from glass-backed source was measured using the 4.0 ns laser by varying the motorized $\lambda / 2$ waveplate such that the peakpositive hydrophone voltage matched with the glass-backed source measured using the $2.6 \mathrm{~ns}$ laser. The settings for each of the four motorized $\lambda / 2$ waveplate settings were noted and finally measurements were repeated with PDMS-based source.

\section{RESULtS AND DisCUSSION}

\section{A. Thickness of CPN Sources}

At $600 \mathrm{~nm}$ wavelength the average optical absorbance and thickness of CPN sources backed on glass was $2.03 \pm 0.05$ and $23 \pm 2.4 \mu \mathrm{m}$, respectively, which results in $\mu_{\mathrm{a}}(\lambda=600 \mathrm{~nm})$ of $203 \pm 23 \mathrm{~mm}^{-1}$. Using this average value of $\mu_{\mathrm{a}}$, which is expected to be the same for both glass and PDMS-backed sources, the thickness for each PDMS-backed source was estimated. The derived thickness of PDMSbacked sources is shown in Table 1 along with measured values of optical absorbance of glass and PDMS-backed sources and the measured thickness of glass-backed sources.

\section{B. Planarity of LGUS Field}

The $-20 \mathrm{~dB}$ beam-areas of the LGUS field for the two lasers, i.e., 2.6 and $4.0 \mathrm{~ns}$ were found to be 1.36 and $0.94 \mathrm{~cm}^{2}$, respectively (see Fig. 2). Although the beam-area of $4.0 \mathrm{~ns}$ laser is $45 \%$ smaller than the $2.6 \mathrm{~ns}$ laser its effect on spatial averaging was considered negligible. The magnitude of the spatial averaging error can be estimated if both the $-6 \mathrm{~dB}$ beam-width and the effective hydrophone radius at each frequency are known [14]. The average spectral beam-widths up to $100 \mathrm{MHz}$ in two orthogonal directions computed using the raster scan data for 2.6 and $4.0 \mathrm{~ns}$ lasers were 7.4 and $5.1 \mathrm{~mm}$, respectively. The geometrical diameter of UC1604 hydrophone was assumed to be the effective hydrophone diameter at all frequencies. Using these values, the magnitude of the spatial averaging error in the LGUS field of 2.6 and 4.0 ns lasers was 0.1 and $0.2 \%$, respectively. 


\section{Measured LGUS Response from CPN Sources}

Example pressure-time series and their spectra for glass and PDMS-backed sources for both 2.6 and $4.0 \mathrm{~ns}$ lasers are shown in Fig. 3. The peak-positive pressures from the glassbacked source is $5 \mathrm{MPa}$, for both pulse durations for an applied laser energy of $16 \mathrm{~mJ}$. However, for the PDMSbacked source the pressures are 3.3 and $3.1 \mathrm{MPa}$ for 2.6 and 4.0 ns lasers, respectively. There are two things to note. First, the pressures for the glass and PDMS-backed sources are not the same, and second, there is a small decrease in pressure with increased laser pulse duration for the PDMSbacked source. These two effects were observed at all energy levels over repeated measurement sets, and for the two pairs of glass and PDMS-backed sources tested in this study.

The differences in the observed acoustic pressures between glass and PDMS-backed sources may be explained with the following argument. The optical absorption coefficient of the CPN film comprising of $2.5 \mathrm{wt} \%$ CNT dispersed in PDMS was previously measured as $167 \mathrm{~mm}^{-1}$ at $1064 \mathrm{~nm}$ [7]. This gives a stress confinement time, $\tau_{\mathrm{s}}=\left(c_{0} \cdot \mu_{\mathrm{a}}\right)^{-1}$ of $5.7 \mathrm{~ns}$ when using the sound-speed of optically clear cured PDMS, $1050 \mathrm{~m} \cdot \mathrm{s}^{-1}$, for $c_{0}$. For efficient laser generation of ultrasound, the laser pulse duration should be short compared to the stress confinement time. Failing to meet this criterion would mean that the pressure wave will start to leave the optical absorption region while pressure is still being added by the laser pulse. The pressure 'spreads out' rather than 'builds up', and therefore the maximum amplitude decreases as the laser pulse duration increases. This effect is seen in the case of PDMS-backed source where the measured pressures were 3.3 and $3.1 \mathrm{MPa}$ for 2.6 and $4.0 \mathrm{~ns}$ lasers, respectively. The big difference in amplitude of $52 \%$ and $61 \%$ in case of glass-backed source relative to PDMS-backed source for 2.6 and $4.0 \mathrm{~ns}$ lasers, respectively is caused by the presence of acoustically strong reflective backing provided by the glass medium. The acoustic impedances of cured PDMS and borosilicate glass calculated using available sound-speeds and mass densities [7] are 1.01 and 12.7 MRayl, respectively, which gives a pressure amplitude reflection coefficient of 0.85 at the interface from PDMS into glass. Consider two acoustically identical half-spaces, the right-hand one of which is optically absorbing. A plane wave pulse of light, short enough for stress confinement, is incident normally through the transparent medium onto the absorbing medium, generating an initial pressure profile that resembles the profile of the absorbed optical energy [15]. In this 1D, stress confined, case, the initial acoustic pressure will divide into two equal parts, one propagating to the left the other to the right. Now, if the transparent half-space were replaced by a material with a higher acoustic impedance, glass say, a

TABLE I. OPticAl AbSORBANCE AND THICKNESSES OF CPN Films.

\begin{tabular}{ccccc}
\hline \multicolumn{2}{l}{ Optical absorbance, $A(\lambda=600 \mathrm{~nm})$} & & \multicolumn{2}{c}{ CPN film thickness, $d[\mu \mathrm{m}]$} \\
\cline { 1 - 2 } \cline { 4 - 5 } Glass-backed & PDMS-backed & & Glass-backed & PDMS-backed \\
\hline $2.04(0.02)$ & $2.36(0.02)$ & & $24(1.3)$ & $27(3.1)$ \\
$2.07(0.03)$ & $2.55(0.04)$ & & $25(3.2)$ & $29(3.4)$ \\
$1.95(0.02)$ & $2.43(0.13)$ & & $20(2.3)$ & $27(3.5)$ \\
$2.03(0.01)$ & $1.23(0.04)$ & & $23(1.6)$ & $14(1.7)$ \\
\hline
\end{tabular}

Parenthetical entries represent one standard deviation in the measured and/or derived values.

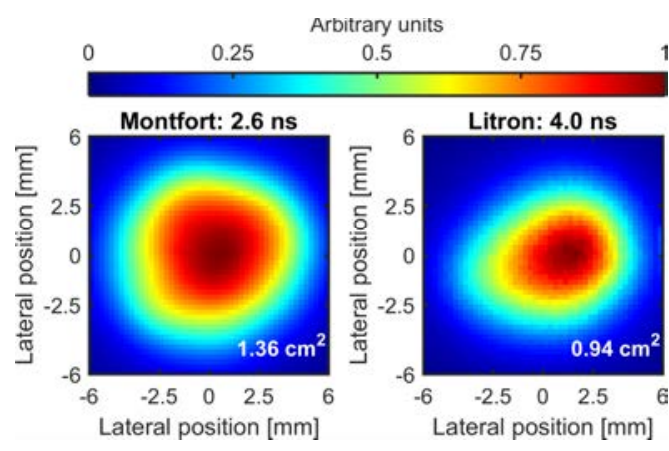

Fig. 2. Raster scan of the LGUS field generated from a glass-backed source and measured using a $0.2 \mathrm{~mm}$ diameter membrane hydrophone. The peak hydrophone voltages recorded from the raster scans were rescaled between $(0,1)$. The areas denoted within each raster scan were estimated at the $-20 \mathrm{~dB}$ of peak value.

proportion of the left-going wave will be reflected back, immediately following the right-going wave.

Therefore, the pressure wave generated from a CPN source backed on a perfectly acoustically reflecting material will be twice that of a source with a backing acoustically matched to the CPN source. This means the bandwidth of the laser generated ultrasound from a PDMS-backed source will be approximately twice that of a glass-backed source without affecting the wave's amplitude. For the case of a glassbacked source in the absence of stress confinement, the leftgoing wave will be reflected by the glass back to the right during the continued optical deposition of heat and consequent build-up of pressure. The total acoustic pressure reached is therefore higher than in the absence of the reflection. In the case of PDMS-backed sources, the pressure amplitude is lower since no reflections are present.

The bandwidths $(-20 \mathrm{~dB})$ from the glass-backed source for applied laser energies of 6, 9, 12 and $16 \mathrm{~mJ}$ were 53, 55, 58 and $60 \mathrm{MHz}$, respectively. The difference in bandwidth between the two lasers was small $(<1 \%)$. The bandwidths obtained from PDMS-backed sources were found to be higher by 7 to $12 \%$ compared to glass-backed sources and were also dependent on laser pulse durations (see Fig.4). Since the acoustic pressure amplitude from the glass-backed
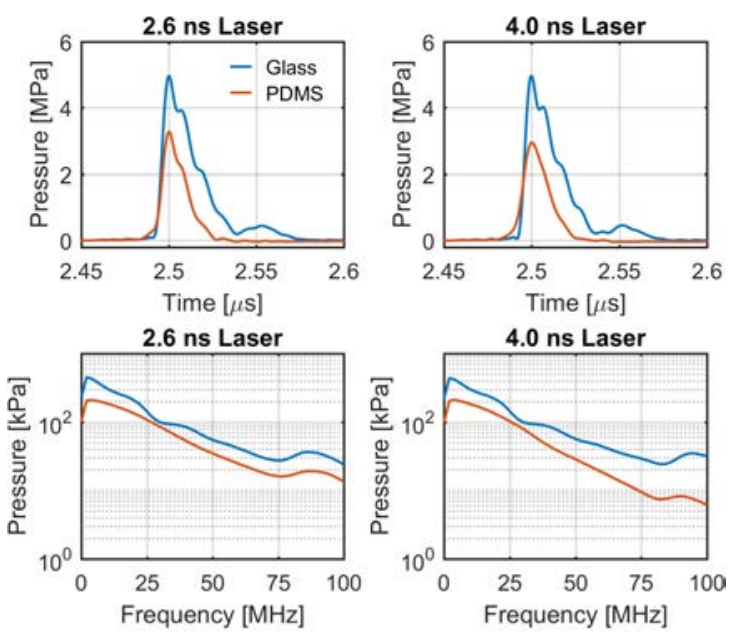

Fig. 3. The pressure time-series and the corresponding spectra for a glass-backed and a PDMS-backed source for an applied laser energy of $16 \mathrm{~mJ}$ for the two lasers. The thicknesses of glass and PDMSbacked sources were 27 and $25 \mu \mathrm{m}$, respectively. 


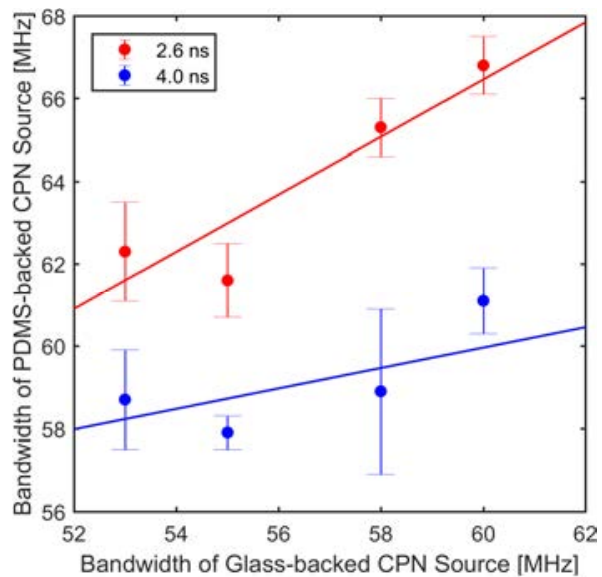

Fig. 4. Plot showing the $-20 \mathrm{~dB}$ bandwidths of a PDMS-backed source obtained using 2.6 and $4.0 \mathrm{~ns}$ lasers for an applied laser energy of $6,9,12$ and $16 \mathrm{~mJ}$ plotted against the average $-20 \mathrm{~dB}$ bandwidths using the same two lasers obtained from glass-backed sources. The error bars represent random uncertainty in the meausrement at two standard deviations. The solid lines are linear regression fits to the measured data.

source is at least $50 \%$ higher than the PDMS-backed source, the increased amplitude resulted in significant wave steepening due to nonlinear propagation [7]. In an ideal case i.e., if the duration of the laser pulse were significantly shorter than the two laser pulse durations, and in the absence of nonlinear propagation, the bandwidths from PDMSbacked source would be double compared to glass-backed source.

\section{CONCLUSIONS}

The effect of backing on carbon-polymer nanocomposites (CPN) comprising of 2.5 weight percent carbon nanotubes dispersed in polydimethylsiloxane (PDMS) backed on glass and clear cured PDMS backing were assessed as sources of laser generation of ultrasound using two pulsed lasers of durations 2.6 and $4.0 \mathrm{~ns}$. A PDMS backing is acoustically closely matched to the CPN whereas a glass backing is acoustically highly reflective. When stress confinement is not satisfied, as with the CPN sources tested in this study, there is a significant difference in the characteristics of the acoustic waves generated from glass and PDMS-backed sources. The peak acoustic pressure amplitude generated from the glass-backed source was 5.0 $\mathrm{MPa}$ for both lasers for an applied laser energy of $16 \mathrm{~mJ}$ whereas it was 3.3 and 3.1 MPa for 2.6 and $4.0 \mathrm{~ns}$ lasers, respectively at the same laser energy. The increase in pressure observed from the glass-backed sources was due to the waves reflected back into the $\mathrm{CPN}$ at the interface of glass-CPN, providing an additional contribution to the pressure build-up to that supplied by heating of laser pulse alone. The difference in bandwidths is not significant compared to amplitudes since the frequency content of an ultrasound pulse is affected by nonlinear propagation, which is dependent on both amplitude and propagation distance. Since the pressure amplitude from the glass-backed source was at least a factor of 1.5 higher than the PDMS-backed source for a given laser energy, the higher amplitude resulted in significant wave steepening, increasing the bandwidth of the pulse.

\section{ACKNOWLEDGMENT}

The UK Department for Business, Energy \& Industrial Strategy's funding of the National Measurement System is gratefully acknowledged. S.R. thanks National Physical Laboratory (NPL) for supporting his $\mathrm{PhD}$ with University College London. B.T.C. and B.E.T. acknowledge the European Union's Horizon 2020 research and innovation program H2020 ICT 2016-2017 under Grant agreement No. 732411, which is an initiative of the Photonics Public Private Partnership.

\section{REFERENCES}

[1] E. J. Alles et al., "Video-rate all-optical ultrasound imaging," Biomed. Opt. Express, vol. 9, no. 8, pp. 3481-3494, 2018.

[2] E. Vannacci, S. Granchi, L. Belsito, A. Roncaglia, and E. Biagi, "Wide bandwidth fiber-optic ultrasound probe in MOMS technology: Preliminary signal processing results," Ultrasonics, vol. 75, pp. 164-173, 2017.

[3] B. Y. Hsieh, S. L. Chen, T. Ling, L. J. Guo, and P. C. Li, "Alloptical scanhead for ultrasound and photoacoustic imagingImaging mode switching by dichroic filtering," Photoacoustics, vol. 2, no. 1, pp. 39-46, 2014.

[4] H. W. Baac, J. Frampton, J. G. Ok, S. Takayama, and L. J. Guo, "Localized micro-scale disruption of cells using laser-generated focused ultrasound," Journal of Biophotonics, vol. 6, no. 11-12. pp. 905-910, 2013.

[5] H. W. Baac et al., "Carbon-nanotube optoacoustic lens for focused ultrasound generation and high-precision targeted therapy.," Sci. Rep., vol. 2, p. 989, Jan. 2012.

[6] M. D. Brown, D. I. Nikitichev, B. E. Treeby, and B. T. Cox, "Generating arbitrary ultrasound fields with tailored optoacoustic surface profiles," Appl. Phys. Lett., vol. 110, no. 9, pp. 1-5, 2017. S. Rajagopal, T. Sainsbury, B. E. Treeby, and B. T. Cox, "Laser generated ultrasound sources using carbon-polymer nanocomposites for high frequency metrology," J. Acoust. Soc. Am., vol. 144, no. 2, pp. 584-597, Aug. 2018.

[8] S. Noimark et al., "Polydimethylsiloxane Composites for Optical Ultrasound Generation and Multimodality Imaging," Adv. Funct. Mater., vol. 1704919, pp. 1-16, 2018.

[9] S.-L. Chen, "Review of Laser-Generated Ultrasound Transmitters and Their Applications to All-Optical Ultrasound Transducers and Imaging," Appl. Sci., vol. 7, no. 1, p. 25, 2016.

[10] 62127-2:2007+AMD1:2013: Amendment 1 - Ultrasonics Hydrophones - Part 2: Calibration for ultrasonic fields up to 40 $\mathrm{MHz}$. International Electrotechnical Commission, Geneva, 2013.

[11] M. a Eddings, M. a Johnson, and B. K. Gale, "Determining the optimal PDMS-PDMS bonding technique for microfluidic devices," J. Micromechanics Microengineering, vol. 18, no. 6, p. 067001, 2008.

[12] P. N. Gélat, R. C. Preston, and A. Hurrell, "A theoretical model describing the transfer characteristics of a membrane hydrophone and validation," Ultrasonics, vol. 43, no. 5, pp. 331-341, 2005.

[13] A. M. Hurrell and S. Rajagopal, "The practicalities of obtaining and using hydrophone calibration data to derive pressure waveforms," IEEE Trans. Ultrason. Ferroelectr. Freq. Control, vol. 64, no. 1, pp. 126-140, 2016.

[14] B. Zeqiri and A. D. Bond, "The Influence of Wave-Form Distortion on Hydrophone Spatial-Averaging Corrections Theory and Measurement," J. Acoust. Soc. Am., vol. 92, no. 4, pp. 1809-1821, 1992.

[15] L. Wang, Ed., Photoacoustic Imaging and Spectroscopy, vol. 144. CRC Press, 2009. 